\title{
PRODUCTS OF BAIRE SPACES
}

\author{
PAUL E. COHEN
}

\begin{abstract}
Only the usual axioms of set theory are needed to prove the existence of a Baire space whose square is not a Baire space.
\end{abstract}

Assuming the continuum hypothesis $(\mathrm{CH})$, Oxtoby [9] constructed a Baire space whose square is not Baire. We will show in this paper that the assumption of $\mathrm{CH}$ is unnecessary. Such results are greatly enhanced by Krom [5], who showed that if there is such an example, then there is also a metric example.

Remarks of the referee were instrumental in making the main result of this paper an absolute one rather than one of relative consistency. In particular, the author was not aware of the forcing technique of $\$ 2$. Comments on this paper by Franklin Tall were also of great help to the author.

1. Baire spaces and forcing. Suppose $\mathscr{P}=\langle P, \leqq\rangle$ is a partially ordered structure. $\mathcal{P}$ may be regarded as a topological space where the initial segments of $\mathcal{P}$ generate a basis. If $\mathcal{P}$ and $\mathcal{Q}$ are partially ordered sets, then the Cartesian product $P \times Q$ may be partially ordered pointwise to obtain a partially ordered set $\mathscr{P} \times \mathcal{2}$. It is easily seen that $\mathscr{P} \times \mathcal{2}$, considered as a topological space, is homeomorphic to the product of topological spaces $\mathscr{P}$ and 2 .

A topological space is said to be Baire if any countable intersection of its dense open sets is dense. If the space is derived from a partially ordered set as above, then we note that any such countable intersection is necessarily open.

Two elements of a partially ordered set will be called compatible if they have a common predecessor. A partially ordered structure $\mathscr{P}=\langle\boldsymbol{P}$, $\leqq$ will be called fine if for every $p, q \in P$, either

(1) $q \leqq p$ or

(2) there is an $r \leqq q$ which is incompatible with $p$.

Suppose $\pi$ is a countable standard transitive model of Zermelo-Fraenkel set theory (ZFC) and $\mathscr{P}, \mathcal{Q} \in \mathscr{N}$ are partially ordered sets. We collect below some well-known facts.

LEMMA 1.0. If $\mathcal{P}$ is fine, then the following statements are equivalent.

(a) $\mathscr{P}$ is Baire in $\Re$.

(b) Whenever $G$ is $\mathscr{P}$-generic over $\mathfrak{N}$ and $f \in \mathfrak{N}[G]$ is an ordinal valued function with domain $\omega$, then $f \in \mathfrak{R}$.

Received by the editors June 13, 1975.

AMS (MOS) subject classifications (1970). Primary 02K25, 04A30; Secondary 08A10, 54B10, $54 \mathrm{G} 20$.

Key words and phrases. Baire category, forcing, continuum hypothesis. 
Proof. For a proof that (a) implies (b) the reader may consult [11]. This implication does not depend upon being fine. The example of the negative integers, however, shows that some assumption about $\mathcal{P}$ is necessary for the converse.

To see that (b) implies (a), work in $\Re$. Suppose that $\mathscr{P}$ is not Baire: there must be a decreasing sequence $\left\langle\Delta_{n} \mid n \in \omega\right\rangle$ of dense open subsets of $\mathscr{P}$ and some $q_{0} \in \mathscr{P}$ with no predecessor in $\cap\left\{\Delta_{n} \mid n \in \omega\right\}$. We may assume that for each $n$ there is a set $\left\{p_{\alpha}^{n} \mid \alpha<\kappa_{n}\right\}$ of pairwise incompatible elements of $\mathscr{P}$ such that $\Delta_{n}=\left\{r \in P \mid\left(\exists \alpha<\kappa_{n}\right)\left[r \leqq p_{\alpha}^{n}\right]\right\}$.

Choose a term $\tau$ of the forcing language such that $\Vdash \tau: \omega \rightarrow O n$ and such that for $n \in \omega$ and $\alpha<\kappa_{n}, p_{\alpha}^{n} \Vdash \tau(n)=\alpha$. Suppose some $q_{1} \leqq q_{0}$ decides $\tau(n)$ for all $n$. Then there is some $n_{0}$ such that $q_{1} \notin \Delta_{n_{0}}$ and some $\alpha$ such that $q_{1} \Vdash \tau\left(n_{0}\right)=\alpha$. Clearly $q_{1} p_{\alpha}^{n_{0}}$ and, since $\mathcal{P}$ is fine, there is an $r \leqq q_{1}$ which is incompatible with $p_{\alpha}^{n_{0}}$ (and so $r$ is compatible with some $p_{\beta}^{n_{0}}$ ).

LEMMA 1.1. The following statements are equivalent.

(a) $G \times H$ is $\mathscr{P} \times 2$-generic over $\mathfrak{N}$.

(b) $G$ is $\mathscr{P}$-generic over $\Re$ and $H$ is 2 -generic over $\Re[G]$.

Proof. See [10, p. 367].

LEMMA 1.2. If 9 and 2 are fine then the following are equivalent.

(a) $\mathcal{P} \times \mathscr{2}$ is Baire in $\Re$.

(b) $\mathscr{P}$ is Baire in $\Re$, and whenever $G$ is $\mathscr{P}$-generic over $\Re$, then 2 is Baire in IR $[G]$.

Proof. This is an immediate consequence of Lemmas 1.0 and 1.1.

REMARK. Lemma 1.2 says that if $\mathscr{P}$ and $\mathcal{Q}$ are Baire and fine but $\mathscr{P} \times \mathscr{2}$ is not Baire, then with nonzero probability (with respect to $\mathcal{P}$ ), $\mathcal{Q}$ is not Baire. Forcing with $\mathcal{P}$ must therefore create a new countable collection of dense open subsets of 2 without generating any new countable sequences in 2 (or any other set of $\Re)$. Notice that this is impossible if either $\mathscr{P}$ or $\mathcal{L}$ is countably closed (i.e., decreasing $\omega$ sequences have a lower bound) in $\mathscr{N}$.

2. Stationary sets. A set $S \subseteq \omega_{1}$ is said to be stationary if $S$ intersects every closed unbounded (c. u. b.) subset of $\omega_{1}$. The following lemma ensures the existence of many stationary sets.

LEMMA 2.0. If $\kappa$ is an uncountable cardinal, then every stationary subset of $\kappa$ is the union of $\mathrm{cf}(\kappa)$ mutually disjoint stationary sets.

Proof. See [11].

The following forcing conditions were related to the author by the referee.

LEMMA 2.1. If $S$ is a stationary subset of $\omega_{1}$, then there is a fine set $\mathscr{P}_{S}$ of Baire forcing conditions which adds a closed unbounded subset of $S$.

Proof. $P_{S}$ is defined as the set of all countable subsets of $S$ which are closed in $\omega_{1}$. If $p, q \in P_{S}$, then $p \leqq q$ means that $p$ is an end extension of $q$ (i.e., $p \supseteq q$ and $(p \backslash q) \cap(\cup q)$ is empty).

Since each $p \in P_{S}$ has a largest member, $\mathscr{P}_{S}$ is clearly fine. If $\mathscr{P}_{S}$ adds no new ordinal valued functions on $\omega$, then it clearly adds a closed unbounded subset of $S$, and by Lemma 1.0 it is Baire as well. 
Suppose for some term $\tau$ of the forcing language and some $p \in \mathscr{P}_{S}, p \Vdash \tau$ : $\omega \rightarrow$ On. In order to finish our proof we need only show there is a $q \leqq p$ such that $q \Vdash \tau \in V$.

Recursively define $R_{\alpha}, \eta_{\alpha}$ such that:

(1) $\left\langle\eta_{\alpha} \mid \alpha<\omega_{1}\right\rangle$ is a continuously increasing sequence of countable ordinals.

(2) $\left\langle R_{\alpha} \mid \alpha<\omega_{1}\right\rangle$ is a continuously increasing sequence of countable subsets of $P_{S}$.

(3) $R_{\alpha} \subseteq\left\{r \in P_{S} \mid r \subseteq \eta_{\alpha} \wedge r \leqq p\right\}$.

(4) $\left(\forall r \in R_{\alpha}\right)(\forall n \in \omega)\left(\exists s \in R_{\alpha+1}\right)\left[s<r \wedge s \subseteq \eta_{\alpha} \wedge s \| \tau(n)\right]$.

Since $S$ is stationary there is a limit ordinal $\alpha$ such that $\eta_{\alpha} \in S$. Choose an increasing sequence $\left\langle\alpha_{n} \mid n \in \omega\right\rangle$ with limit $\alpha$ and a sequence $r_{0}>r_{1}>\cdots$ such that $r_{n} \in R_{\alpha_{n}}, r_{n+1} \subseteq \eta_{\alpha_{n}}$ and $r_{n} \| \tau(n)$. Let $q=\left\{\eta_{\alpha}\right\} \cup\left(\cup\left\{r_{n} \mid n \in \omega\right\}\right)$. Since $q \| \tau(n)$ for all $n, q \Vdash \tau \in V$.

LEMMA 2.2. If $S_{1}$ and $S_{2}$ are disjoint stationary subsets of $\omega_{1}$, then $\mathscr{P}_{S_{1}} \times \mathscr{P}_{S_{2}}$ is not Baire.

Proof. Suppose that $\mathscr{P}_{S_{1}} \times \mathcal{P}_{S_{2}}$ is Baire. Then the closed unbounded set added in the extension by $\mathscr{P}_{S_{1}}$ remains closed and unbounded in the extension by ${ }^{P_{S_{1}}} \times \mathscr{P}_{S_{2}}$. Likewise there is a closed unbounded subset of $S_{2}$ which is added by $\mathscr{P}_{S_{1}} \times \mathscr{P}_{S_{2}}$. Since it follows that in this extension there are two disjoint c.u.b. subsets of $\omega_{1}$, our assumption must be false.

THEOREM 2.3. There are two Baire spaces whose product is not a Baire space. In fact, there is a Baire space whose square is not Baire.

PRoof. $\mathscr{P}_{S_{1}}$ and $\mathscr{P}_{S_{2}}$ of Lemma 2.2 serve for the first statement while their direct sum (disjoint union) serves for the second.

In the next section we consider the problem: Is there a Baire space whose square is nowhere Baire?

3. The Suslin conjecture. Solovay and Tennenbaum [12] showed the relative consistency of an axiom which has come to be known as Martin's axiom. Martin's axiom was invented in order to show the relative consistency of the Suslin conjecture [8] which says that there are no Suslin trees.

Martin's axiom can be thought of as a weak version of the continuum hypothesis. For example, Tall [14] remarks that Oxtoby's example of a Baire space whose square is not Baire works if Martin's axiom (and not necessarily $\mathrm{CH})$ is assumed. It is interesting then that a Suslin tree may be a Baire space whose square is nowhere Baire.

If $\alpha$ is an ordinal, then denote by $\alpha^{*}$ the order type of the reversed ordering on $\alpha$. A tree is a partially ordered set $\mathcal{T}=\langle T, \leqq\rangle$ such that for every $x \in T$ there is an ordinal $|x|$ such that $\{y \in T \mid y>x\}$ has order type $|x|^{*}$. Notice that two points of a tree are comparable iff they are compatible. The height of a tree $\mathcal{T}$ is defined as $\cup\{|t| \mid t \in T\}$. A Suslin tree is a tree of height $\omega_{1}$ which satisfies the countable chain condition (c.c.c.) and in which all chains are countable.

The following fact is well known.

Lemma 3.0. A Suslin tree $\mathcal{T}$ is Baire. 
Proof. If $\Delta$ is a dense open subset of $T$ then for some $\alpha<\omega_{1}, \Delta$ $\supseteq\{x|| x \mid>\alpha\}$.

REMARK 3.1. A Suslin tree cannot be countably closed, for this would allow an uncountable chain to be defined.

REMARK 3.2. Although a Suslin tree $\mathcal{T}$ satisfies c.c.c., $\mathfrak{T} \times \mathcal{T}$ does not. $\left\{\left\langle x, x^{\prime}\right\rangle \in T \times T \mid x \neq x^{\prime} \wedge(\forall y \in T)\left[y>x \leftrightarrow y>x^{\prime}\right]\right\}$ has power $\aleph_{1}$, and is pairwise incompatible.

The situation here is similar to Lemma 1.2. If $\mathscr{N}$ is a countable standard transitive model of ZFC, $\mathcal{T}$ is a Suslin tree in $\mathscr{T}$ and $G$ is $\mathcal{T}$-generic over $\mathscr{T}$, then although $\mathcal{T}$ is c.c.c. in $\Re$ it is not c.c.c. in $\Re[G]$ : for each $x \in G$ which has two incompatible predecessors, choose one predecessor $x^{\prime} \notin G$. The set of these $x^{\prime}$ is pairwise incompatible and of power $\boldsymbol{\kappa}_{1}$.

REMARK 3.3. Lemma 3.0 has a partial converse [6]: If there is a Baire partially ordered set $\varphi \rho$ with c.c.c. and no atoms ( $p$ is an atom if there are no incompatible $q, q^{\prime} \leqq p$ ), then there is a Suslin tree.

To see this, recursively define $T=\cup_{\alpha<\omega_{1}} T_{\alpha} \subseteq P$ where

(1) each $T_{\alpha}$ is a maximal pairwise incompatible subset of $\mathcal{P}$;

(2) if $\alpha<\beta$ and $t_{\alpha} \in T_{\alpha}$ then $\left\{t \in T_{\beta} \mid t \leqq t_{\alpha}\right\}$ has at least two elements and is a maximal pairwise incompatible subset of $\left\{p \in P \mid p<t_{\alpha}\right\}$.

REMARK 3.4. If a tree $\mathcal{T}=\langle T, \leqq\rangle$ is c.c.c. and such that every $t \in T$ has at least two incompatible predecessors then $\mathcal{T}$ is Suslin. For suppose

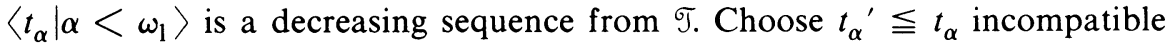
with $t_{\alpha+1}$. Then $\left\langle t_{\alpha}{ }^{\prime} \mid \alpha<\alpha_{1}\right\rangle$ is a sequence of pairwise incompatible elements of $\mathscr{T}$, which by assumption cannot exist.

Jensen and Johnsbråten [4] showed that there is a Suslin tree $\mathcal{T}$ in $L$ with the property that $\sigma$ has at most one uncountable branch (maximal chain) in the real world. The referee has remarked that $\mathcal{T}$ has this same property in a model of $V=L[G]$ where $G$ is a set of Cohen generic reals (over $L$ ).

If $\mathfrak{T}$ is a countable standard transitive model of $\mathrm{ZFC}+V=L, \mathcal{T} \in \mathfrak{N}$ is the tree mentioned above, and $G \times H$ is $\mathscr{T} \times \mathcal{T}$ generic over $\mathfrak{T}$, then $\omega_{1}$ $\neq \omega_{1}^{L}$ in $\Re[G \times H]$. From Lemma 1.0 it follows that although $\sigma$ is Baire in $\mathfrak{T}, \mathcal{T} \times \mathcal{T}$ is not.

We will show that if $\mathscr{K}$ is a countable standard transitive model of $\mathrm{ZFC}$, then there is a Cohen extension $\mathfrak{K}$ of $\mathfrak{R}$ (using conditions similar to those of Tennenbaum [12]) and a Suslin tree $\mathcal{T} \in \mathcal{N}$ which enjoys the properties (relativized to $\Re$ ) of the tree mentioned above. $\mathcal{T}=\langle T$, $\leqq$ will be such that

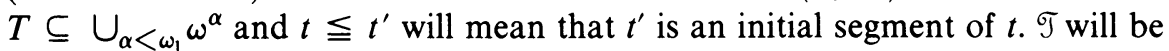
so constructed that if $t, t^{\prime}$ are not comparable and $t_{\alpha}, t_{\alpha}{ }^{\prime}$ are both defined, then $\left\langle\left|t_{\beta}-t_{\beta}^{\prime}\right| \mid \beta \leqq \alpha\right\rangle$ is a sequence, eventually of distinct positive natural numbers.

If two distinct branches of $\mathcal{T}$ with length $\omega_{1}$ are given, then an $\omega_{1}$ sequence of distinct positive natural numbers may be defined. Thus in any extension $\Re^{\prime} \supseteq \Re$ which preserves $\omega_{1}$, there is at most one uncountable branch.

Let $\mathfrak{N}$ be a countable standard transitive model ZFC. Define a set $\mathscr{P} \in \mathfrak{N}$ of forcing conditions. A condition is a finite set $U$ such that each $f \in U$ is a function into $\omega$ such that $\operatorname{dom}(f) \subseteq \omega_{1}$ is finite. If $f, f^{\prime} \in U$ then we require

(1) if $f(\alpha)=f^{\prime}(\alpha)$ then $f \uparrow \alpha=f^{\prime} \uparrow \alpha$;

(2) if $f(\alpha) \neq f^{\prime}(\alpha)$ (but both are defined) then $g(\beta)=\left|f(\beta)-f^{\prime}(\beta)\right|$ 
defines a positive valued one-to-one function into $\omega$ on $\{\beta \in \operatorname{dom}(f)$ $\left.\cap \operatorname{dom}\left(f^{\prime}\right) \mid \beta \geqq \alpha\right\}$.

We will say that $U \leqq U^{\prime}$ if for every $f^{\prime} \in U^{\prime}$ there is an $f \in U$ such that $f \supseteq f^{\prime}$.

If $U \in \mathcal{P}$, then define $A_{U}=\cup_{f \in U} \operatorname{dom}(f)$. If $A \subseteq \omega_{1}$, then define $U \uparrow A$ $=\{f \uparrow A \mid f \in U\}$. The following is a special case of a result due to Marczewski [7].

LEMMA 3.5. If $\left\langle U_{\alpha}\right| \alpha\left\langle\omega_{1}\right\rangle$ is a sequence from $\mathscr{P}$ in $\mathscr{T}$, then there is a finite $C \subseteq \omega_{1}$ and a subsequence $\left\langle V_{\alpha} \mid \alpha<\omega_{1}\right\rangle$ such that

$$
\left(\forall \alpha<\beta<\omega_{1}\right)\left[A_{V_{\alpha}} \cap A_{V_{\beta}}=C \wedge V_{\alpha} \uparrow C=V_{\beta} \uparrow C\right]
$$

Proof. If there is a subsequence with $A_{U_{\alpha}}$ constant, then we may, in fact, assume that $\left\langle V_{\alpha}\right| \alpha\left\langle\omega_{1}\right\rangle$ is constant, in which case we are done.

Thus, we may assume that for no subsequence is $A_{U_{\alpha}}$ a constant. With no loss of generality we may assume that for all $\alpha, A_{U_{\alpha}}$ has $n$ elements $\left\{\gamma_{\alpha}^{1}<\cdots<\gamma_{\alpha}^{n}\right\}$. Choose $k \leqq n$ smallest so that $\left\langle\gamma_{\alpha}^{k} \mid \alpha<\omega_{1}\right\rangle$ is not bounded below $\omega_{1}$. We may well assume that $U_{\alpha} \uparrow\left\{\gamma_{\alpha}^{1}, \ldots, \gamma_{\alpha}^{k-1}\right\}$ is constant in $\alpha$ and that $\gamma_{\alpha}^{n}<\gamma_{\beta}^{k}$ whenever $\alpha<\beta<\omega_{1}$. But now the lemma is seen to hold with $C=\left\{\gamma_{0}^{1}, \ldots, \gamma_{0}^{k-1}\right\}$.

LEMMA 3.6. $\mathscr{P}$ is c.c.c. in $\Re$.

Proof. Otherwise there is a sequence $\left\langle V_{\alpha}\right| \alpha\left\langle\omega_{1}\right\rangle$ of incompatible conditions which has the form described in Lemma 3.6. Since, however, $V_{\alpha} \cup V_{\beta}$ $\leqq V_{\alpha}, V_{\beta}$, this is impossible.

Let $G$ be $\mathscr{P}$-generic over $\mathfrak{R}$ and let $G^{*}=\cup G$. If $\alpha<\omega_{1}$ and $n \in \omega$, then let $t_{n}^{\alpha}=\left\{\langle\beta, m\rangle \mid \beta \leqq \alpha \wedge\{\langle\alpha, n\rangle,\langle\beta, m\rangle\} \in G^{*}\right\}$. Then it is easy to see that $\sigma=\langle T, \supseteq\rangle$ is an $\omega_{1}$-tree where $T=\left\{t_{n}^{\alpha} \mid \alpha<\omega_{1} \wedge n<\omega\right\}$. For $\alpha$ $<\omega_{1}$ and $n \in \omega$ let $\tau_{n}^{\alpha}$ be a name in the forcing language for $t_{n}^{\alpha}$.

LEMMA 3.7. $\sigma$ is a fine Suslin tree.

Proof. It is easy to see that every $t \in T$ has two incompatible predecessors and that $\mathcal{T}$ is fine. Thus it suffices to show that $\mathcal{T}$ is c.c.c. (see Remark 3.4).

Suppose that $\sigma$ is a name and $V_{0} \in G$ is such that $V_{0} \Vdash \sigma: \omega_{1} \rightarrow \mathbf{T}$ is an enumeration of pairwise incompatible elements of $\mathcal{T}$. For $\alpha<\omega_{1}$, choose (in গ) $U_{\alpha} \leqq V_{0}$ and $n_{\alpha} \in \omega, \gamma_{\alpha} \in \omega_{1}$ such that $U_{\alpha} \Vdash \sigma(\alpha)=\tau_{n_{\alpha}}^{\gamma_{\alpha}}$. For ease of notation let us write $\tau_{\alpha}$ for $\tau_{n_{\alpha}}^{\gamma_{\alpha}}$.

We may as well assume that for some $f_{\alpha} \in U_{\alpha}, f_{\alpha}\left(\gamma_{\alpha}\right)=n_{\alpha}$. Use Lemma 3.5 to choose a subsequence $\left\langle U_{\alpha_{\beta}} \mid \beta<\omega_{1}\right\rangle$ of $\left\langle U_{\alpha} \mid \alpha<\omega_{1}\right\rangle$. But now

$$
V=\left(U_{\alpha_{0}} \backslash\left\{f_{\alpha_{0}}\right\}\right) \cup\left(U_{\alpha_{1}} \backslash\left\{f_{\alpha_{1}}\right\}\right) \cup\left\{f_{\alpha_{0}} \cup f_{\alpha_{1}}\right\} \leqq U_{\alpha_{0}}, U_{\alpha_{1}}
$$

and $V \Vdash \tau_{\alpha_{0}} \leqq \tau_{\alpha_{1}}$ in contradiction to the choice of $\sigma$ and $V_{0}$.

THEOREM 3.8. In $\mathscr{T}[G]$, $\mathcal{T}$ is a Baire space but $\mathcal{T} \times \mathcal{T}$ is not.

Proof. This is the proof which was outlined after Remark 3.4. The fact that $\mathcal{\sigma}$ is a Baire space follows immediately from Lemmas 3.0 and 3.7. The fact that $\mathfrak{T} \times \mathcal{T}$ is not Baire follows from Lemmas 1.0, 3.7, and the following tact which 
the reader can easily verify. If $\left\langle t_{\alpha}\right| \alpha\left\langle\omega_{1}\right\rangle$ and $\left\langle t_{\alpha}^{\prime} \mid \alpha<\omega_{1}\right\rangle$ are both sequences of comparable elements of $\mathcal{T}$, say with $\operatorname{dom}\left(t_{\alpha}\right)=\operatorname{dom}\left(t_{\alpha}{ }^{\prime}\right)=\alpha$ +1 and $t_{\alpha_{n}} \neq t_{\alpha_{0}}^{\prime}$ (such sequences cannot, of course, be in $\Re$ ), then $\left\langle\left|t_{\alpha}(\alpha)-t_{\alpha}^{\prime}(\alpha)\right|\right| \alpha_{0} \leqq \alpha\left\langle\omega_{1}\right\rangle$ is a sequence of distinct positive integers.

REMARK 3.9. Since $P$ has cardinality $\kappa_{1}$ in $\Re, 2^{\omega}$ is the same in $\Re$ and $\Re[G]$. Thus $\mathrm{CH}$ fails in $\Re[G]$ iff it fails in $\Re$.

\section{BIBLIOGRAPHY}

1. P. J. Cohen, Set theory and the continuum hypothesis, Benjamin, New York and Amsterdam, 1966. MR 38 \#999.

2. T. J. Jech, Lectures in set theory, with particular emphasis on the method of forcing, Lecture Notes in Math., vol. 217, Springer-Verlag, Berlin and New York, 1971. MR 48 \# 105.

3. —, Trees, J. Symbolic Logic 36 (1971), 1-14. MR 44 \#1560.

4. R. B. Jensen and H. Johnsbråten, $A$ new construction of a nonconstructible $\Delta_{3}^{1}$ subset of $\omega$, Univ. of Oslo preprint series, 1973, no. 3.

5. M. R. Krom, Cartesian products of metric Baire spaces, Proc. Amer. Math. Soc. 42 (1974), 588-594. MR 48 \#12457.

6. D. Maharam, Set functions and Souslin's hypothesis, Bull. Amer. Math. Soc. 54 (1948), 587-590. MR 9, 573.

7. E. Marczewski, Séparabilité et multiplication cartésienne des espaces topologiques, Fund. Math. 34 (1947), 127-143. MR 9, 98.

8. E. W. Miller, A note on Souslin's problem, Amer. J. Math. 65 (1943), 673-678. MR 5, 173.

9. J. C. Oxtoby, Cartesian products of Baire spaces, Fund. Math. 49 (1960/61), 157-166. MR 25 \# 4055; 26, p. 1543.

10. J. R. Shoenfield, Unramified forcing, Proc. Sympos. Pure Math., vol 13, Amer. Math. Soc., Providence, R. I., 1971, pp. 357-381. MR 43 \#6079.

11. R. M. Solovay, Real-valued measurable cardinals, Proc Sympos. Pure Math., vol. 13, Amer. Math. Soc., Providence, R. I., 1971, pp. 397-428. MR 45 \#55.

12. R. M. Solovay and S. Tennenbaum, Iterated Cohen extensions and Souslin's problem, Ann. of Math. (2) 94 (1971), 201-245. MR 45 \#3212.

13. M. Souslin, Problème 3, Fund. Math. 1 (1920), 223.

14. F. Tall, An alternative to the continuum hypothesis and its uses in general topology, (unpublished manuscript).

15. G. Takeuti and W. Zaring, Axiomatic set theory, Graduate Texts in Math., vol. 8, SpringerVerlag, New York, 1973.

16. S. Tennenbaum, Souslin's problem, Proc. Nat. Acad. Sci. U.S.A. 59 (1968), 60-63. MR 37 $\# 55$.

Department of Mathematics, Lehigh University, Bethlehem, Pennsylvania 18015 